\title{
1 KRAS phosphorylation regulates cell polarization and tumorigenic properties in
}

2

3

4 Débora Cabot $^{1,2}$, Sònia Brun ${ }^{1,2}$, Noelia Paco ${ }^{1,2}$, Mireia M. Ginesta ${ }^{3}$, Núria Gendrau5 Sanclemente $^{1,8}$, Baraa Abuasaker ${ }^{1,2}$ Triana Ruiz-Fariña ${ }^{1}$, Carles Barceló $^{1,2,9}$, Miriam

6 Cuatrecasas $^{2,4}$, Marta Bosch ${ }^{1,2}$, Carles Rentero ${ }^{1,2}$, Gabriel Pons ${ }^{6}$, Josep M Estanyol ${ }^{1,7}$, Gabriel 7 Capellà $^{3}$, Montserrat Jaumot ${ }^{1,2 *}$, Neus Agell ${ }^{1,2}$.

8

$9{ }^{1}$ Dept. Biomedicina, Facultat de Medicina i Ciències de la Salut, Universitat de Barcelona., 10 Barcelona, Spain.

$11{ }^{2}$ Institut d'Investigacions Biomèdiques August Pi i Sunyer (IDIBAPS), Barcelona, Spain.

$12{ }^{3}$ Hereditary Cancer Program, Translational Research Laboratory, Catalan Institute of Oncology, 13 ICO-IDIBELL, Hospitalet de Llobregat, Barcelona, Spain and Centro de Investigación

14 Biomédica en Red en Cáncer (CIBERONC), Madrid, Spain.

$15{ }^{4}$ Departament de Fonaments Clínics, Facultat de Medicina i Ciències de la Salut, Universitat de 16 Barcelona; Pathology Department and Centro de Investigación Biomédica en Red de 17 Enfermedades Hepáticas y Digestivas (CIBERehd) and Tumor Bank-Biobank, Hospital Clínic, 18 Barcelona, Spain.

${ }^{6}$ Departament de Ciències Fisiològiques, Facultat de Medicina i Ciències de la Salut, Universitat 20 de Barcelona and Institut d'Investigació Biomèdica de Bellvitge (IDIBELL), L'Hospitalet de Llobregat, Barcelona, Spain.

${ }^{7}$ Proteomics Unit, CCiT-UB, University of Barcelona, Barcelona, Spain.

${ }^{8}$ Present address: Program Against Cancer Therapeutic Resistance (ProCURE), Catalan Institute of Oncology, Hospital Duran i Reynals, Institut d'Investigació Biomèdica de Bellvitge (IDIBELL), L'Hospitalet de Llobregat, Barcelona, Spain.

${ }^{9}$ Present address: Institut d'Investigació Sanitària Illes Balears (IdISBa), Palma de Mallorca, Spain.

\section{Keywords}

RAS / CTNNA1 / HNF4G / SERPINE1 / PRSS2 / NEO1

*Corresponding authors:

Neus Agell Jané: neusagell@ub.ub; Montserrat Jaumot Pijoan: mjaumot@ub.edu 


\section{Abstract}

35 Oncogenic mutations of KRAS are found in the most aggressive human tumors, including 36 colorectal cancer. It has been suggested that oncogenic KRAS phosphorylation at Ser181 37 modulates its activity and favors cell transformation. Using non-phosphorylatable (S181A), 38 phosphomimetic (S181D) and phospho/dephosphorylatable (S181) oncogenic KRAS mutants, 39 we analyzed the role of this phosphorylation to the maintenance of tumorigenic properties of 40 colorectal cancer cells. Our data show that the presence of phospho/dephosphorylatable 41 oncogenic KRAS is required for preserving the epithelial organization of colorectal cancer cells 42 in $3 \mathrm{D}$ cultures, and for supporting subcutaneous tumor growth in mice. Interestingly, gene 43 expression differed according to the phosphorylation status of KRAS. In DLD-1 cells, 44 CTNNA1 was only expressed in phospho/dephosphorylatable oncogenic KRAS expressing 45 cells, correlating with cell polarization. Moreover, lack of oncogenic KRAS phosphorylation 46 leaded to changes in expression of genes related to cell invasion, such as SERPINE1, PRSS1,2,3 47 and NEO1, and expression of phosphomimetic oncogenic KRAS resulted in diminished 48 expression of genes involved in enterocyte differentiation, such as HNF4G. Finally, the 49 analysis, in a public data set of human colorectal cancer, of the gene expression signatures 50 associated to phosphomimetic and non-phosphorylatable oncogenic KRAS suggests that this 51 post-translational modification regulates tumor progression in patients. 


\section{Introduction}

53 KRAS is a member of the Ras family of small GTPases. Its wild type form cycles from the 54 inactive (GDP-bound) to the active (GTP-bound) state, responding faithfully to extracellular 55 signals. When GTP-bound, it interacts with effector proteins that activate diverse signal 56 transduction pathways, which in turn regulate processes such as proliferation, survival or 57 differentiation in normal cells, the best studied being the c-RAF/MEK/ERK and PI3K/AKT ${ }^{1}$.

58 All RAS isoforms have a highly conserved globular domain that contains the catalytic lobe and 59 the allosteric lobe; and the non-conserved C-terminal domain, the hypervariable region (HVR), 60 which contains the membrane targeting signals ${ }^{2}$. RAS proteins are irreversibly modified by 61 farnesylation in the cysteine of the C-terminal CAAX sequence. Uniquely, adjacent to this 62 modified aminoacid, KRAS has also a stretch of six contiguous lysines, which promotes an 63 electrostatic interaction with the negatively-charged phosphate groups of phospholipids ${ }^{3}$.

$64 R A S$ is a major oncogenic driver in a variety of tumor types. Oncogenic KRAS mutations are 65 found in the most deadly cancers (pancreatic (91\%), colorectal (CRC, 42\%), and lung (33\%)) $)^{1,4}$. 66 Although oncogenic mutations preserve KRAS in its GTP-bound state, diverse evidences

67 suggest that oncogenic KRAS can be regulated, and so there may be several factors that 68 maintain GTP-bound KRAS in a non-signaling state ${ }^{5-7}$.

69 Non-effector proteins that bind to the HVR or/and the allosteric lobe of KRAS, such as PDE6$70 \delta^{5}$, galectin $3^{8}$, calmodulin $(\mathrm{CaM})^{9-11}, \operatorname{HNRNPA2B} 1^{12}$, nucleophosmin ${ }^{13}$ and $\beta$-catenin ${ }^{14}$, are 71 examples of proteins that can modulate oncogenic KRAS activity s, $\mathrm{s}^{6,15,16}$. Additionally, several 72 post-translational modifications of KRAS such as phosphorylation, ubiquitination or acetylation 73 have been reported to be also able to modulate oncogenic KRAS activity ${ }^{6,16-21}$; among them, 74 phosphorylation at Ser181, within the HVR, is the most studied. We demonstrated that this 75 phosphorylation is regulated by $\mathrm{CaM}$ interaction ${ }^{6}$ and that expression of phosphomimetic mutants of oncogenic KRAS in normal mouse fibroblasts favored activation of downstream

77 signaling, cell transformation, and tumor growth in mouse models ${ }^{6,12,18}$. Our data obtained in 78 DLD-1 cells deleted for the endogenous oncogenic KRAS allele and overexpressing exogenous 79 non-phosphorylatable or phosphorylatable oncogenic KRAS at Ser181 also confirmed the role 
80 of S181 phosphorylation for CRC tumor growth ${ }^{18}$. It has also been described using other

81 cellular models, that KRAS phosphorylation induces apoptosis ${ }^{20}$ and that non-phosphorylated

82 KRAS, by capturing $\mathrm{CaM}$, inhibits the non-canonical $\mathrm{Wnt} / \mathrm{Ca} 2+$ signaling and promotes

83 tumorigenicity ${ }^{19}$. These contrasting results may be due to the use of different cellular models but

84 may also be due to the distinct expression levels of the oncogenic KRAS.

85 Although it is widely accepted that KRAS is a good target for cancer therapy, its inhibition 86 represents a challenge. Interfering with its post-translational modifications such as 87 phosphorylation at Ser181 may open a new therapeutic opportunity, but first, the relevance of 88 this phosphorylation in the maintenance of the tumorigenic properties of established cancer cells 89 must be demonstrated. To this end, we have generated CRC cells expressing different oncogenic 90 KRAS phosphomutants. Our data show that the presence of phospho/dephosphorylatable 91 oncogenic KRAS is essential for maintaining the polarity of the CRC cells and for allowing 92 tumor growth, and interestingly, that the presence of non-phosphorylatable oncogenic KRAS 93 impairs the invasive capacity of cells. Thus, we conclude that CRC cells depend on KRAS 94 phosphorylation at Ser181 to maintain their tumorigenic properties. 
Results

99

100

101

\section{Colorectal cancer cells expressing different oncogenic KRAS phosphomutants show different epithelial morphology in 2D culture}

To study the role of oncogenic KRAS phosphorylation at Ser181 in CRC, DLD-1 cells were used. These cells carry an oncogenic mutant KRAS allele and a wild type (WT) KRAS allele. This cell line was chosen because it depends on the expression of the oncogenic allele of KRAS to fulfill its tumorigenic properties; thus, the isogenic DLD-1 cell line knocked out for the oncogenic KRAS allele (named DLD-1 KO in this paper) does not grow properly under growth factor limiting conditions and does not generate tumors when subcutaneously injected in mice $^{22,23}$. DLD-1 KO cells were transfected to generate clones of cells with recovered expression of oncogenic KRAS, but with different mutations at position 181. Consequently, clones of cells expressing different levels of exogenous oncogenic non-phosphorylatable KRAS (KRAS-S181A), oncogenic phosphomimetic KRAS (KRAS-S181D) or the control oncogenic phospho/dephosphorylatable KRAS (KRAS-S181) were obtained. When examined by phasecontrast microscopy, and regardless of the phosphomutant, all cells with high levels of expression of oncogenic KRAS showed a mesenchymal morphology, while clones expressing oncogenic KRAS at levels like endogenous KRAS maintained an epithelial-like morphology (Fig. 1a and Supplementary Fig. S1a).

To analyze the role of oncogenic KRAS phosphorylation in CRC cells, we chose clones that expressed oncogenic KRAS phosphomutants at levels comparable to those of the endogenous WT KRAS (Fig. 1a, upper panel). Interestingly, although in 2D cultures all clones showed an epithelial-like morphology, clear differences between them were observed. Similar to the original DLD-1 (Supplementary Fig. S1b), cells expressing oncogenic KRAS-S181 were able to form compact clusters, in which the boundaries between the cells were barely perceptible (Fig. 1a, bottom panel). Conversely, this type of cell organization was not observed with the oncogenic KRAS-S181A and KRAS-S181D clones. Furthermore, oncogenic KRAS-S181D 
cells were rounder than the rest. In conclusion, the phosphorylation status of oncogenic KRAS is relevant for establishing a specific cell morphology in this CRC cell line.

126

127

Oncogenic KRAS expression induces cell proliferation and modulates ERK and AKT activation regardless of the Ser181 phosphorylation status of KRAS

To study the relevance of KRAS phosphorylation in cell viability under serum-limiting conditions, cell growth at $0.1 \%$ Fetal Bovine Serum (FBS) was determined for DLD-1, DLD-1 $\mathrm{KO}$ and the different oncogenic KRAS phosphomutants (Fig. 1b). As expected, DLD-1 KO cells grew less than DLD- $1^{22,23}$ and cells expressing oncogenic KRAS-S181 recovered the ability to grow under serum-limiting conditions. Both oncogenic KRAS-S181A and KRASS181D clones grew significantly more than DLD-1 KO cells and similarly to oncogenic KRASS181 and DLD-1, indicating that growth under starvation was independent of the phosphorylation status of KRAS. In agreement with the proliferation data, the levels of P-AKT and P-ERK in the different oncogenic KRAS phosphomutants were similar to those in DLD-1

Phosphorylation status of oncogenic KRAS differentially regulates gene expression in colorectal cancer cells

To better understand the phenotypes observed in DLD-1 cells expressing the different oncogenic KRAS phosphomutants, we analyzed and compared their gene expression. Clustering analysis of the differentially expressed genes demonstrated distinct expression patterns between the phosphomutant clones. The greatest differences were found between oncogenic KRASS181A and KRAS-S181D clones (Fig. 2a, b, Supplementary Table S1 and Supplementary Fig. 
152 S2a). Although few, the existence of differentially expressed genes between oncogenic KRAS-

153 S181A and KRAS-S181 clones indicates that a proportion of KRAS is phosphorylated, and

154 plays a role in the regulation of gene expression (Fig. 2b, cand Supplementary Table S1). Levels

155 of oncogenic KRAS-S181 phosphorylation were indirectly estimated by measuring its affinity

156 to $\mathrm{CaM}^{9,19}$. Two out of the three oncogenic KRAS-S181 clones tested presented a reduced

157 binding to CaM, indirectly corroborating oncogenic KRAS phosphorylation in those S181

158 clones (Supplementary Fig. S2b).

159 Genes related to enterocyte differentiation such as HNF4G, HEPH, MUC13, and UGT1A $24-27$

160 were particularly downregulated in oncogenic KRAS-S181D cells compared to both oncogenic

161 KRAS-S181 and KRAS-S181A (Fig. 2c and Supplementary Fig. S2a), suggesting that KRAS

162 phosphorylation induces a de-differentiation program. Changes in HNF4G expression were

163 corroborated by qPCR and Western Blot (WB) (Fig. 2d and Supplementary Fig. S2c, d).

164 Furthermore, GSEA indicated that the expression signature of oncogenic KRAS-S181D versus

165 oncogenic KRAS-S181A and -S181 expressing cells is similar to that of DLD-1 cells with

166 upregulated LEF1 (Fig. 2e), a gene related to the WNT signaling pathway and pluripotency ${ }^{28}$.

167 TRIB2, recently proposed as an oncogene in $\mathrm{CRC}^{29}$, showed increased expression in cells with

168 the phosphomimetic mutant, as demonstrated by qPCR (Fig. 2d and Supplementary Fig. S2d).

169 Genes differentially expressed in oncogenic KRAS-S181A vs KRAS-S181 clones are involved

170 in cell invasion and vascular co-option ${ }^{30,31}$. Specifically, PRSS1,2,3 (coding for different

171 isoforms of trypsin), and SERPINE1 (coding for PAI-I) are among the genes whose expression

172 was specifically inhibited more than 2-fold when oncogenic KRAS could not be phosphorylated

173 (Fig. 2c). Furthermore, NEO1 (codifying for neogenin 1), a suppressor of wound-healing

174 response ${ }^{32}$, is the only gene whose expression was significantly increased more than 2-fold in

175 oncogenic KRAS-S181A-expressing cells (Fig. 2c). Decreased mRNA levels of SERPINE1 and

176 PRSS2 were corroborated by qPCR (Fig. 2d and Supplementary Fig. S2d), and increased levels

177 of neogenin 1 were corroborated by WB (Supplementary Fig. S2c).

178 CTNNA1 was the only gene whose expression decreased in either S181A or S181D oncogenic

179 KRAS-expressing cells compared with KRAS-S181 (Fig. 2c). This result was confirmed by 
qPCR and WB (Fig. 2d, Supplementary Fig. S2d and Fig. 3a). Interestingly, the product of CTNNA1, $\alpha$-E-catenin, is involved in cell-to-cell adhesion, a characteristic that we found to be impaired in cells expressing either the S181A or the S181D mutants of oncogenic KRAS (Fig. 1a, bottom panel).

\section{Phosphorylation status of KRAS affects both organization of cells growing in 3D cultures} and cell invasion capacity

Since $\alpha$-E-catenin is involved in cell-to-cell adhesion and polarization, we aimed to analyze a possible impact of oncogenic KRAS phosphorylation status in cell growth and organization in 3-Dimensional (3D) culture.

When grown in soft agar, although there was variability between clones, all oncogenic KRAS phosphomutants had a significantly higher capacity to form colonies than DLD-1 KO cells (Supplementary Fig. S3a). Interestingly, the morphology of cell colonies expressing phospho/dephosphorylatable oncogenic KRAS differed from those expressing either oncogenic KRAS-S181A or KRAS-S181D. While colonies of cells expressing the S181 oncogene were compact spheres, colonies of non-phosphorylatable or phosphomimetic mutant cells were noncompact and flatter, with well-distinguished limits between cells (Supplementary Fig. S3b). Growth in Matrigel-based 3D cultures was then analyzed. DLD-1 KO cells formed smaller cellular aggregates than the cell lines expressing oncogenic KRAS (Fig. 3b and Supplementary Fig. S3c). Interestingly, while the three oncogenic KRAS-S181 clones formed large, compact spheroidal and organized structures with a central hollow, all oncogenic KRAS-S181A or KRAS-S181D clones assembled into disorganized and branched "grape-like" aggregates (Fig. 3b). Cells expressing the phosphomimetic oncogenic KRAS were the ones less aggregated. Immunofluorescence analysis showed that cells expressing oncogenic KRAS-S181 formed an epithelial structure of polarized cells with a central lumen. E-cadherin positive contacts between cells could be observed, and polymerized actin was localized in the apical cortex (near the lumen) resembling a structure containing microvilli (Fig. 3c). Finally, $\alpha 6$-integrin was confined 
to the basal part of the cells (Fig. 3c). In contrast, all these markers indicated that cells

208 expressing phosphomimetic or non-phosphorylatable oncogenic KRAS were not polarized.

209 These data suggest that the lack of a phosphorylation-dephosphorylation cycle of oncogenic

210 KRAS interfered with the polarization of the cells and, consequently, with the formation of an 211 organized epithelial structure. Finally, immunofluorescence analysis of Matrigel cultures

212 demonstrated that, in cells expressing the phospho/dephosphorylatable KRAS mutant, $\alpha$-E-

213 catenin was localized at the plasma membrane and mainly in the areas of contact between the

214 cells while it was undetectable in the phosphomimetic and non-phosphorylatble mutant cells.

215 (Fig. 3c).

216 The fact that, as mentioned above (Fig. 2b, c and Supplementary Table S1), the few 217 differentially expressed genes in oncogenic KRAS-S181A vs KRAS-S181 clones were related 218 to cell invasion, prompted us to study the impact of the lack of KRAS phosphorylation on cell 219 invasiveness capacity. To test it, we used SW480 cells harboring oncogenic mutations in the 220 two KRAS alleles and being a CRC cell line more prone to invade than DLD-1 cells. 221 Interestingly, the single mutation S181A in one of the KRAS alleles leaded to a diminished 222 expression of SERPINE1 as demonstrated by qPCR (Fig. 4a) and WB (Fig. 4b), and to a 223 reduced invasive capacity (Fig. 4c). Lack of $\alpha$-E-catenin and increased neogenin-1 expression 224 were also corroborated by either qPCR or WB in these cells (Fig. 4a, b).

226 Tumor growth is impaired in cells expressing oncogenic non-phosphorylatable or 227 phosphomimetic KRAS

228 To test whether the phosphorylation status of oncogenic KRAS was also relevant to support 229 tumor growth in CRC cells, DLD-1 KO cells and oncogenic KRAS phosphomutants were 230 subcutaneously injected into nude mice, and tumor growth was monitored over time (Fig. 5a, b 231 and Supplementary Fig. S4a). As expected, DLD-1 KO cells generated very few tumors, which 232 were almost imperceptible macroscopically. In accordance with the previous data obtained with 233 immortalized mouse fibroblasts ${ }^{18}$, CRC cells expressing oncogenic KRAS-S181 developed 234 subcutaneous tumors, while tumor growth was clearly impaired in oncogenic KRAS-S181A 
cells. But surprisingly, tumor growth was also reduced in CRC cells expressing oncogenic KRAS-S181D (Fig. 5b and Supplementary Fig. S4a).

237 Interestingly, the histological comparation of the tumors revealed differences in cell organization similar to those observed in cells growing in 3D cultures. While oncogenic KRASS181-expressing cells formed well-organized epithelial structures around blood vessels, the oncogenic KRAS-S181A and KRAS-S181D tumors were less differentiated, composed by cells that were poorly organized around smaller or collapsed blood vessels (Fig. 5c and Supplementary Fig. S4b). This lack of organization around the blood vessels could be one of the causes of impaired growth of tumors derived from KRAS-S181D and S181A-expressing epithelial CRC cells. In fact, WB analysis showed that CA-IX expression was higher in tumors derived from these cells, indicating higher levels of hypoxia (Supplementary Fig. S4c). Furthermore, IHC analysis showed a different distribution of CA-IX signal in the area around the blood vessels (Supplementary Fig. S4b). In the oncogenic KRAS-S181 ones we observed a clear hypoxic negative area around the blood vessels, followed by a strong positive region containing CA-IX positive hypoxic cells, while in the oncogenic KRAS-S181A and -S181Dderived tumors, hypoxic cells were found much near to the blood vessels. Lack of $\alpha$-E-catenin in tumors expressing phosphomimetic or non-phosphorylatable oncogenic KRAS was confirmed (Fig. 5d). A reduction in the amount of trypsin protein was also observed in non-phosphorylatable mutant-derived tumors (Fig. 5d).

254 Histological analysis showed that tumors generated by all KRAS phosphomutants, presented areas composed of apoptotic and necrotic cells which were TUNEL positive, as well as regions of high cell proliferation which were Ki-67 positive (Supplementary Fig. S4b). The mitotic count in the proliferating areas of all tumors was similar (Supplementary Fig. S4b, d). Finally, the effect of oncogenic KRAS phosphorylation on c-RAF/MEK/ERK and PI3K/AKT signaling pathways in the tumors was similar to that observed in 2D cultures (Fig. 5e). Furthermore, no correlation was observed between tumor growth and the activation status of these two signal transduction pathways. 
262 To generalize the need of KRAS phosphorylation and dephosphorylation for cell polarity and 263 tumor growth, the study was extended to HCT116 cells, a CRC cell line that also has oncogenic 264 KRAS and an epithelial morphology. HCT116 cells KO for oncogenic KRAS were transfected 265 with the different oncogenic KRAS phosphomutants. Similar to DLD-1 cells, the only clones 266 that were able to form polarized compact organoid-like structures in 3D (analyzed either by 267 phase contrast microscopy or immunofluorescence) were the ones expressing oncogenic KRASS181 (Supplementary Fig. S5a). Changes of HNFG4 and Neo1 expression observed in the DLD-1 clones, were confirmed in HCT116 cell line (Supplementary Fig. S5b). In contrast, $\alpha$-Ecatenin did not follow the same expression pattern in HCT116 cells than in DLD-1 cells, suggesting that the reduced expression of this protein observed in DLD-1 cells might be a consequence of lack of cell polarization more than the primary cause (Supplementary Fig. S5b). Interestingly, tumor grow upon subcutaneous injection of these cells in mice was also reduced in clones expressing oncogenic KRAS-S181A and KRAS-S181D, compared to the clone expressing the oncogenic KRAS-S181, which is the only one that can be subjected to the phosphorylation-dephosphorylation cycle (Supplementary Fig. S5c).

277 Finally, we analyzed if expression of higher levels of oncogenic KRAS could revert the 278 decreased tumor growth observed in cells expressing either non-phosphorylatable or 279 phosphomimetic oncogenic KRAS. As shown in Supplementary Fig. S6a, and in agreement with our previous published data ${ }^{18}$, DLD-1 cells with high overexpression of oncogenic KRASS181A had highly impaired the ability to produce subcutaneous tumors. Interestingly, now we show that (in contrast that what occurs in fibroblasts) this ability was also impaired in epithelial cells overexpressing high levels of oncogenic KRAS-S181D (Supplementary Fig. S6a). Remarkably, this occurs independently that, in agreement with our previously published data, in 2D-serum restricted conditions, phosphomimetic mutants grew better than nonphosphorylatable mutants (Supplementary Fig. S6b). Thus, suggesting that, independently of the levels of oncogenic KRAS expression, phospho/dephosphorylation cycle of KRAS is essential to support tumor growth, but not growth in 2D cultures. Interestingly, the clones overexpressing phospho/dephosphorylatable oncogenic KRAS were again the only ones 
showing some capacity to form polarized organoid-like structures when grown in Matrigel

291 (Supplementary Fig. S6c).

292

293

KRAS phosphorylation/dephosphorylation gene expression signature in human colorectal tumors

295 To analyze the relevance of KRAS phosphorylation status in human CRC development, the 296 expression of genes belonging to the KRAS-S181A signature (differentially expressed between 297 oncogenic KRAS-S181A and KRAS-S181) and genes belonging to the KRAS-S181D signature 298 (differentially expressed between oncogenic KRAS-S181D and KRAS-S181) was examined in 299 a public data set of CRC samples (GSE39582) $)^{33}$. Firstly, in general, a positive correlation was 300 observed in the tumors when comparing separately genes upregulated or downregulated belonging to the same signature (either KRAS-S181A or KRAS-S181D), while a negative correlation was observed when comparing upregulated and repressed genes within a signature (Fig. 6a). Importantly, a negative correlation was observed when comparing KRAS-S181A versus KRAS-S181D signatures. All this supported the hypothesis that these genes are cocompared to tumor samples, we noticed that gene expression profiles of the tumors were more similar to the KRAS phosphorylation signature than to the non-phosphorylated one (Fig. 6b). Finally, patients with tumors overexpressing NEO1 (overexpressed in KRAS-S181A vs -S181) or with tumors with low levels of SERPINE1 (downregulated in KRAS-S181A vs -S181) had longer DFS, while patients with tumors with low expression of HNG4G (downregulated in KRAS-S181D vs -S181) or high expression of ID4 (overexpressed in KRAS-S181D vs -S181) had a shorter DFS (Fig. 6c). 


\section{Discussion}

315 Data presented here indicate that modification of the Ser181 phosphorylation status of

316 oncogenic KRAS in CRC cells strongly impacts on the behavior of these cells: the presence of a

317 phospho/dephosphorylatable residue at position 181 in oncogenic KRAS is essential for cell polarization and aggregation and for facilitating subcutaneous tumor growth; and, cells expressing a non-phosphorylatable or a phosphomimetic amino acid at this position show differential expression of genes with a prominent role in oncogenesis.

321 The role of phosphorylation of Ser181 in the HVR of KRAS is still controversial. The studies 322 performed to date have mainly been done in non-transformed cell lines as a model, and so what has been analyzed is the contribution of S181 phosphorylation in initial cell transformation ${ }^{18,20,34}$. In the present work we used DLD-1 cells, which are oncogenic KRASdependent and have been shown to be a good model for the study of $\mathrm{CRC}^{35,36}$. This has allowed us to investigate the role of oncogenic KRAS phosphorylation in maintaining the tumoral properties of cancer cells. An important point of our research is that we exogenously expressed diverse oncogenic KRAS-S181 phosphomutants in a modified DLD-1 cell line with a deletion of the endogenous oncogenic KRAS allele (DLD-1 KO), so the endogenous oncogene did not mask the impact of the exogenous phosphomutants. Furthermore, in contrast to our previous study $^{18}$, for the main part of the current work, we chose cell clones expressing levels of exogenous oncogenic KRAS similar to those of endogenous WT KRAS, so avoiding possible additional effects due only to oncogenic KRAS overexpression, such as the induction of a mesenchymal phenotype.

Important for our work is that all phosphomutant constructs produced functional oncogenic KRAS proteins, since all recovered the growth of DLD-1 KO cells at serum-starving conditions or in soft agar. This also indicated that the signaling pathways activated by KRAS that allow cells to survive under those conditions are independent of the phosphorylation status of KRAS.

339 Accordingly, a similar impact of all phosphomutants was observed on the last effectors of the 340 main KRAS signaling pathways c-RAF/MEK/ERK and PI3K/AKT. 
341 Although no significant differences were perceived regarding in vitro cell growth, major 342 changes in cellular aggregation and organization were observed between cells expressing the 343 different oncogenic KRAS phosphomutants, the differences being more evident in cells grown 344 in Matrigel. Cells expressing a phospho/dephosphorylatable oncogenic KRAS were the only 345 ones able to form glandular-like structures with polarized cells. This was also observed in 346 HCT116 cells and in DLD-1 cells with overexpression of oncogenic KRAS. One could argue 347 that the mutation of serine to aspartic acid does not properly mimic phosphorylation, but this is 348 unlikely to be the case, since we find a high number of genes differentially expressed between 349 oncogenic KRAS-S181A and -S181D cells and in all previous publications a different 350 phenotype was observed between cells expressing these phosphophomutants $6,18-20$. 351 Additionally, S181D mutant had a reduced binding to $\mathrm{CaM}$ indicating that, at least in this 352 aspect, it was mimicking KRAS phosphorylation. Thus, we hypothesize that the presence of 353 both phosphorylated and dephosphorylated oncogenic KRAS is essential to achieve cell 354 polarity. Interestingly, atypical PKC activity located specifically in the apical domain of 355 epithelial cells is required for proper maintenance of cell polarization ${ }^{37}$. Accordingly, 356 phosphorylated KRAS could also be located in the apical domain and dephosphorylated KRAS 357 in the basolateral domain participating in cell polarization (Supplementary Fig. 7). Lack of cell 358 aggregation and polarization in both oncogenic KRAS-S181A and -S181D-expressing DLD-1 359 clones correlated with a reduced expression of CTNNA1, which codes for $\alpha$-E-catenin. Since $\alpha$ 360 E-catenin facilitates actin attachments at the adherent junctions ${ }^{38}$, the lack of $\alpha$-E-catenin may 361 contribute to the loss of intercellular adhesion. But, because the same pattern of expression of $\alpha$ 362 E-catenin was not observed in HCT116 cells, decreased levels of this protein in DLD-1 cells 363 may be a consequence and not the primary cause of their inability to organize a well-polarized 364 epithelium in Matrigel.

365 An important conclusion of the transcriptomic analysis is that the phosphorylation status of 366 KRAS at Ser181 modulates the expression of specific genes in these CRC cells. Besides, it can 
be stated that at least a proportion of oncogenic KRAS is being phosphorylated, which we have indirectly confirmed by CaM pull-down.

Gene expression differences between oncogenic KRAS-S181 and KRAS-S181A cells were mainly found in genes involved in cell migration, invasion and metastases ${ }^{30,31}$, indicating that the cells expressing the non-phosphorylatable oncogenic KRAS might have low invasion capacity. These gene expression changes were corroborated in another CRC cell line, SW480, in which we introduced a S181A mutation in one of the oncogenic KRAS alleles. Most interestingly, these mutant cells displayed less ability to invade. Although further experiments are needed, from our results we suggest that oncogenic KRAS phosphorylation enhances cell invasion.

Additionally, the specific differences in gene expression induced by the phosphomimetic mutant, imply that phosphorylation of KRAS promotes an undifferentiated cellular state related to cancer progression. The reduced expression of genes such as $H N F 4 G^{27}, H E P H, U G T 1 A$ and $M U C 13^{24-26}$, and the GSEA data associate KRAS phosphorylation with pluripotency ${ }^{28}$.

Notably, expression correlation analysis, in a cohort of human CRC, between genes belonging to the different signatures strongly supports the hypothesis that gene expression is also regulated by KRAS phosphorylation in human tumors.

While all cells expressing the different phosphomutants of oncogenic KRAS were able to grow in 2D and 3D cultures, subcutaneous tumor growth, independently of the levels of expression of the phosphomutant, was strongly impaired in KRAS-S181A and in KRAS-S181D-expressing cells. Results obtained with the non-phosphorylatable mutant agreed completely with our previous observations ${ }^{18}$, but based on the gene expression data and in $2 \mathrm{D}$ culture results, it was surprising that the phosphomimetic mutant did not support tumor growth. Interestingly, lack of tumor growth correlated (independently of the levels of oncogene expression) with the inability to form polarized organoid-like structures in Matrigel. The poorly differentiated histological morphology and the lack of a well-organized perivascular organization observed in oncogenic 
KRAS-S181D and KRAS-S181A tumors may reflect the 3D culture findings (Supplementary

394 Fig. 7) and may preclude tumor nutrition and oxygenation (in agreement with the observed CA-

395 IX expression), and consequently tumor growth. We propose that, as was the case with 396 Matrigel, a phosphorylation/dephosphorylation cycle is necessary to polarize and organize the 397 cells around blood vessels. Interestingly, cell polarity in CRCs is disrupted but not completely $398 \operatorname{lost}^{39}$. Thus, a selective pressure to maintain certain cell polarity may exist in colorectal tumors. 399 Accordingly, $\alpha$-E-catenin has an essential role in intestinal adenoma formation ${ }^{40}$ and together 400 with other components of the cadherin complex is considered an obligatory haploinsufficient tumor suppressor in intestinal neoplasia ${ }^{41}$. The need of cell polarization for CRC tumor growth, may explain the differences observed regarding the ability of mouse fibroblast transfected with oncogenic KRAS-S181D to generate subcutaneous tumors ${ }^{18}$. Based on the findings presented here one might think that inducing either complete KRAS phosphorylation or dephosphorylation would be a good therapeutic strategy: both PKC inhibitors and activators 406 have been shown to reduce tumor growth induced by oncogenic KRAS ${ }^{18,42}$. Nevertheless, present data related to cell invasion and differentiation, together with our previous observations

408 in mouse fibroblasts ${ }^{6,12,18}$, suggest that inhibiting KRAS phosphorylation would be safer. Most 409 importantly, analysis of the public data indicates that gene expression in human CRC is more 410 similar to the phosphomimetic than to the non-phosphorylatable oncogenic KRAS signature, 411 supporting the hypothesis that phosphorylation is important for human CRC development, and 412 that consequently its inhibition would be a good therapeutic strategy.

413 We conclude that CRC cells depend on KRAS phosphorylation cycle at Ser181 to maintain 414 their tumorigenic properties. Specific interference with this modification or with its downstream 415 signaling may be an appropriate therapy. 
418 Cell lines and culture. DLD-1 (KRAS ${ }^{\mathrm{WT} / \mathrm{G} 13 \mathrm{D}}$ ) (clone V15, \#HD PAR-086) and HCT116

419 (KRAS $\left.^{\mathrm{WT} / \mathrm{G} 13 \mathrm{D}}\right)($ \#HD PAR-007) colorectal adenocarcinoma cell lines, and DLD-1 and HCT116 420 knockouts of mutant KRAS allele, DLD-1 KO (KRAS ${ }^{\mathrm{WT} /}$ ) (clone D-WT7, \#HD105-002) and 421 HCT116 KO (KRAS ${ }^{\mathrm{WT} /}$ ) (clone HAF1 (v154), \#HD 104-008) were obtained from Horizon 422 Discovery Ltd. (Cambridge, UK). DLD-1 KO and HCT116 KO mutant clones stably 423 expressing HA-KRAS-G12V-S181, HA-KRAS-G12V-S181A, or HA-KRAS-G12V-S181D 424 were generated by transfecting DLD-1 KO and HCT116 KO cells with the specific HA-KRAS425 G12V plasmids as indicated in Supplementary Methods. SW480 cells with one oncogenic 426 KRAS allele containing the S181A mutation was generated by single guide wild-type Cas9427 based CRISPR technology ${ }^{43}$ (see details in supplementary methods). DLD-1 and DLD-1 KO 428 cells were grown in DMEM-HAM's F12 (1:1), and SW480 in DMEM. In all cases medium was 429 supplemented as previously described ${ }^{18}$. Cells were tested one per month for mycoplasma 430 contamination.

Cell growth and Proliferation assays, Cell invasion Assay, and Sample lysis and Western blotting are detailed in Supplementary Methods and supplementary table S2

434

435

3-Dimensional (3D) cell culture. 3D on-top Matrigel assay was performed as in ref. ${ }^{44}$. For 436 details and also for soft agar colony formation assay see Supplementary Methods.

437

Immunofluorescence for 3D cell culture. Organoid-like structures of growing cells were fixed following option $\mathrm{C}$ of the protocol for whole-culture fixation ${ }^{44}$. See detailed in Supplementary Methods.

441

442 CaM-Sepharose Pull-down Assays were performed as previously described ${ }^{9}$. 443 
444 Tumor generation in mice. Subcutaneous tumors were generated as previously described ${ }^{18}$.

445 See details in Supplementary Methods.

446 All mouse experiments were performed in accordance with protocols approved by the Animal

447 Care and Use Committee of ICO-IDIBELL Hospitalet de Llobregat (Barcelona, Spain). For

448 tumor histology and histochemistry see Supplementary Methods. Antibodies and reagents used

449 are listed in Supplementary Table S3.

450

451

Microarrays and gene expression analysis. See Supplementary Methods and references ${ }^{29,45-50}$ and Supplementary Table S4

453

Dunnett's Multiple Comparisons Tests; and considered when $P<0.05$.

458

Data and code availability. The datasets generated during the current study are available in the GEO database repository: https://www.ncbi.nlm.nih.gov/geo/query/acc.cgi?acc=GSE176276

\section{Acknowledgements}

463 We thank the personnel of the Advanced Microscopy Unit of CCiT-UB (Campus Clinic) for the 464 help in setting up image acquisition and analysis.

465 This work was supported by grants from the Ministerio de Economia y Competitividad and co466 funded by FEDER funds -a way to build Europe- ( SAF2016-76239-R and PID2019467 105483RB-I00 to NA, SAF2015-68016-R to GC), CIBERONC and the Governement of 468 Catalonia (grants 2017SGR1282 and PERIS SLT002/16/0037), and from Instituto de Salud 469 Carlos III (grant PI17/01304 to MC); a FPU fellowship from Ministerio de Educación, Cultura y 470 Deporte for DC, a FI fellowship from Generalitat de Catalunya for NP, and a Predoc-UB from 
471 University of Barcelona to BA; CR is supported by the Serra Húnter Program (Generalitat de 472 Catalunya).

473

474 Competing Interests

475 Authors declare there is not any competing financial interests in relation to the work described.

476

477 Supplementary information is available at Oncogene's website. 


\section{References}

4791 Malumbres M, Barbacid M. RAS oncogenes: the first 30 years. Nat Rev Cancer 2003; 3: $480 \quad 459-65$.

4812 Bourne HR, Sanders DA, McCormick F. The GTPase superfamily: conserved structure $482 \quad$ and molecular mechanism. Nature 1991; 349: 117-127.

4833 Hancock JF, Magee AI, Childs JE, Marshall CJ. All ras proteins are polyisoprenylated $484 \quad$ but only some are palmitoylated. Cell 1989; 57: 1167-77.

4854 Simanshu DK, Nissley D V., McCormick F. RAS Proteins and Their Regulators in $486 \quad$ Human Disease. Cell 2017; 170: 17-33.

4875 Chandra A, Grecco HE, Pisupati V, Perera D, Cassidy L, Skoulidis F et al. The GDI-like 488 solubilizing factor PDE $\delta$ sustains the spatial organization and signalling of Ras family $489 \quad$ proteins. Nat Cell Biol 2012; 14: 148-58.

4906 Alvarez-Moya B, López-Alcalá C, Drosten M, Bachs O, Agell N. K-Ras4B

491 phosphorylation at Ser181 is inhibited by calmodulin and modulates K-Ras activity and 492 function. Oncogene 2010; 29: 5911-22.

4937 Stephen AG, Esposito D, Bagni RG, McCormick F. Dragging ras back in the ring.

$494 \quad$ Cancer Cell 2014; 25: 272-281.

4958 Shalom-Feuerstein R, Plowman SJ, Rotblat B, Ariotti N, Tian T, Hancock JF et al. K-ras 496 nanoclustering is subverted by overexpression of the scaffold protein galectin-3. Cancer $497 \quad$ Res 2008; 68: 6608-16.

4989 Lopez-Alcalá C, Alvarez-Moya B, Villalonga P, Calvo M, Bachs O, Agell N.

499 Identification of essential interacting elements in K-Ras/calmodulin binding and its role $500 \quad$ in K-Ras localization. J Biol Chem 2008; 283: 10621-31.

50110 Garrido E, Lázaro J, Jaumot M, Agell N, Rubio-Martinez J. Modeling and subtleties of 502 K-Ras and Calmodulin interaction. PLoS Comput Biol 2018; 14: 1-19. 
11 Villalonga P, López-Alcalá C, Bosch M, Chiloeches A, Rocamora N, Gil J et al.

504 Calmodulin binds to K-Ras, but not to H- or N-Ras, and modulates its downstream

$505 \quad$ signaling. Mol Cell Biol 2001; 21: 7345-54.

50612 Barceló C, Etchin J, Mansour MR, Sanda T, Ginesta MM, Sanchez-Arévalo Lobo VJ et

507

508 al. Ribonucleoprotein HNRNPA2B1 interacts with and regulates oncogenic KRAS in pancreatic ductal adenocarcinoma cells. Gastroenterology 2014; 147: 882-892.e8.

13 Inder KL, Lau C, Loo D, Chaudhary N, Goodall A, Martin S et al. Nucleophosmin and nucleolin regulate K-Ras plasma membrane interactions and MAPK signal transduction. J Biol Chem 2009; 284: 28410-9.

14 Lee S, Jeong W, Cho Y, Cha P, Yoon J, Ro EJ et al. $\beta$ Catenin RAS interaction serves as a molecular switch for RAS degradation via GSK3ß. EMBO Rep 2018; : e46060.

15 Villalonga P, López-Alcalá C, Chiloeches A, Gil J, Marais R, Bachs O et al. Calmodulin prevents activation of Ras by PKC in 3T3 fibroblasts. J Biol Chem 2002; 277: 3792937935.

16 Barceló C, Paco N, Beckett AJ, Alvarez-Moya B, Garrido E, Gelabert M et al. Oncogenic K-ras segregates at spatially distinct plasma membrane signaling platforms according to its phosphorylation status. J Cell Sci 2013; 126: 4553-9.

17 Yang MH, Nickerson S, Kim ET, Liot C, Laurent G, Spang R et al. Regulation of RAS oncogenicity by acetylation. Proc Natl Acad Sci U S A 2012; 109: 10843-8.

18 Barcelo C, Paco N, Morell M, Alvarez-Moya B, Bota-Rabassedas N, Jaumot M et al. Phosphorylation at Ser-181 of oncogenic KRAS is required for tumor growth. Cancer Res 2014; 74: 1190-1199.

19 Wang MT, Holderfield M, Galeas J, Delrosario R, To MD, Balmain A et al. K-Ras Promotes Tumorigenicity through Suppression of Non-canonical Wnt Signaling. Cell 2015; 163: 1237-1251. 
Bivona TG, Quatela SE, Bodemann BO, Ahearn IM, Soskis MJ, Mor A et al. PKC

529 regulates a farnesyl-electrostatic switch on K-Ras that promotes its association with BclXL on mitochondria and induces apoptosis. Mol Cell 2006; 21: 481-93. Ubiquitination of K-Ras enhances activation and facilitates binding to select downstream effectors. Sci Signal 2011; 4: ra13.

Vartanian S, Bentley C, Brauer MJ, Li L, Shirasawa S, Sasazuki T et al. Identification of mutant K-Ras-dependent phenotypes using a panel of isogenic cell lines. J Biol Chem 2013; 288: 2403-2413.

Shirasawa S, Furuse M, Yokoyama N, Sasazuki T. Altered growth of human colon cancer cell lines disrupted at activated Ki-ras. Science (80- ) 1993; 260: 85 LP - 88.

Brookes MJ, Hughes S, Turner FE, Reynolds G, Sharma N, Ismail T et al. Modulation UDP-glucuronosyltransferase (UGT) genes and cancer risk. Drug Metab Rev 2016; 48: $47-69$.

Maher DM, Gupta BK, Nagata S, Jaggi M, Chauhan SC. Mucin 13: Structure, function, and potential roles in cancer pathogenesis. Mol Cancer Res 2011; 9: 531-537. V, Furlan C et al. Integrative multi omics analysis of intestinal organoid differentiation. Mol Syst Biol 2018; 14: e8227. in cancer, as a biomarker for prognosis and a target for treatment. Am J Cancer Res 2017; 7: 1389-1406. 
in colorectal cancer by blocking cellular senescence through AP4/p21 signaling. Mol

30 Yamamoto H, Iku S, Adachi Y, Imsumran A, Taniguchi H, Nosho K et al. Association

of trypsin expression with tumour progression and matrilysin expression in human colorectal cancer. J Pathol 2003; 199: 176-184.

Li S, Wei X, He J, Tian X, Yuan S, Sun L. Plasminogen activator inhibitor-1 in cancer research. Biomed Pharmacother 2018; 105: 83-94.

56032 Chaturvedi V, Fournier-Level A, Cooper HM, Murray MJ. Loss of Neogenin1 in human 561 colorectal carcinoma cells causes a partial EMT and wound-healing response. Sci Rep $562 \quad 2019 ; 9: 1-15$.

Marisa L, de Reyniès A, Duval A, Selves J, Gaub MP, Vescovo L et al. Gene Expression Classification of Colon Cancer into Molecular Subtypes: Characterization, Validation, and Prognostic Value. PLoS Med 2013; 10: e1001453.

Yin N, Liu Y, Khoor A, Wang X, Thompson EA, Leitges M et al. Protein Kinase Ci and Wnt/ $\beta$-Catenin Signaling: Alternative Pathways to Kras/Trp53-Driven Lung Adenocarcinoma. Cancer Cell 2019; 36: 156-167.e7. cancer cell lines are representative models of the main molecular subtypes of primary cancer. Cancer Res 2014; 74: 3238-3247.

573

57537 Román-Fernández A, Bryant DM. Complex Polarity: Building Multicellular Tissues 576 Through Apical Membrane Traffic. Traffic 2016; 17: 1244-1261. omics of 34 colorectal cancer cell lines - a resource for biomedical studies. Mol Cancer 2017; 16: 1-16. Maiden SL, Hardin J. The secret life of $\alpha$-catenin: Moonlighting in morphogenesis. $J$ 
Cell Biol 2011; 195: 543-552.

57939 Compton CC. Colorectal carcinoma: Diagnostic, prognostic, and molecular features.

$580 \quad$ Mod Pathol 2003; 16: 376-388.

58140 Shibata H, Takano H, Ito M, Shioya H, Hirota M, Matsumoto H et al. Alpha-Catenin is 582 essential in intestinal adenoma formation. Proc Natl Acad Sci 2007; 104: 18199-18204.

58341 Short SP, Kondo J, Smalley-Freed WG, Takeda H, Dohn MR, Powell AE et al. p120-

584 Catenin is an obligate haploinsufficient tumor suppressor in intestinal neoplasia. J Clin

$585 \quad$ Invest 2017; 127: 4462-4476.

58642 Elia AEH, Wang DC, Willis NA, Boardman AP, Hajdu I, Adeyemi RO et al. RFWD3-

587 Dependent Ubiquitination of RPA Regulates Repair at Stalled Replication Forks. Mol

$588 \quad$ Cell 2015; 60: 280-293.

58943 Ran FA, Hsu PD, Wright J, Agarwala V, Scott DA, Zhang F. Genome engineering using $590 \quad$ the CRISPR-Cas9 system. Nat Protoc 2013; 8: 2281-2308.

59144 Lee GY, Kenny PA, Lee EH, Bissell MJ. Three-dimensional culture models of normal $592 \quad$ and malignant breast epithelial cells. Nat Methods 2007; 4: 359-365.

59345 Gogarten SM, Bhangale T, Conomos MP, Laurie CA, McHugh CP, Painter I et al. 594 GWASTools: An R/Bioconductor package for quality control and analysis of genome$595 \quad$ wide association studies. Bioinformatics 2012; 28: 3329-3331.

59646 Subramanian A, Tamayo P, Mootha VK, Mukherjee S, Ebert BL, Gillette MA et al. 597 Gene set enrichment analysis: A knowledge-based approach for interpreting genome$598 \quad$ wide expression profiles. Proc Natl Acad Sci U S A 2005; 102: 15545-50.

59947 Cortazar AR, Torrano V, Martín-Martín N, Caro-Maldonado A, Camacho L, Hermanova 600 I et al. Cancertool: A visualization and representation interface to exploit cancer 601 datasets. Cancer Res 2018; 78: 6320-6328.

60248 Livak KJ, Schmittgen TD. Analysis of relative gene expression data using real-time 
60449 Humphries BA, Buschhaus JM, Chen YC, Haley HR, Qyli T, Chiang B et al.

605 Plasminogen activator inhibitor 1 (PAI1) promotes actin cytoskeleton reorganization and

606 glycolytic metabolism in triple-negative breast cancer. Mol Cancer Res 2019; 17: 1142-

607 1154.

60850 Wang J, Zhang J, Xu L, Zheng Y, Ling D, Yang Z. Expression of HNF4G and its 609 potential functions in lung cancer. Oncotarget 2018; 9: 18018-18028.

610 
612 Fig. 1 Stable expression of oncogenic KRAS phosphomutants induce differential cell 613 morphology. a WB analysis showing the clones of DLD-1 KO (KRAS ${ }^{\mathrm{WT} /}$ ) with an exogenous expression of KRAS-G12V-S181 (S181), KRAS-G12V-S181A (S181A) and KRAS-G12V-

615 S181D (S181D) similar to the endogenous level of KRAS (numbers indicate different clones) 616 (upper panel). Phase-contrast images of KRAS phosphomutants cell clones. All scale bars, 50 $617 \mu \mathrm{m}$ (bottom panel). b $5 \times 10^{3}$ DLD-1 KO $\left(\mathrm{KRAS}^{\mathrm{WT} /}\right.$ ) cells stably expressing either KRASG12V-S181, -S181A, or -S181D were cultured under serum-limiting (0.1\% FBS) conditions for 48 hours to evaluate cell survival by MTT. A cell viability ratio was obtained for each clone. Mean \pm SEM of four independent experiments is shown. Significant differences were assessed using one-way ANOVA and Dunnett's Multiple Comparisons Tests compared to DLD-1 KO $\left({ }^{*}\right.$-value $<0.05, \quad * *$ p-value $<0.01, \quad * * *$ p-value $<0.001, \quad * * * *$ p-value $\left.<0.0001\right) . \quad$ c DLD-1 KO $\left(\mathrm{KRAS}^{\mathrm{WT} /}\right.$ ) cells expressing KRAS-G12V phosphomutants were cultured in absence of serum ( $0 \%$ FBS) for 24 hours and total lysates from the different cell clones were analyzed by WB to detect the indicated proteins. Lamin B and Gap120 were used as loading controls of phosphoproteins. *Gap120 was used as loading controls of total proteins.

Fig. 2 Status of oncogenic KRAS phosphorylation at Ser181 has an impact in genes expression. a Average linkage WPGMA Clustering of proves and clones that had a significantly different expression (FDR $<0.01$ (False Discovery Rate)) in at least one of the conditions (S181; S181A or S181D). Intensities (Log2) were normalized for each gene. b Differentially expressed probes were pooled in genes to determine the number of genes differentially expressed. Number of genes (upper graph) and Venn diagram (lower graph) of differentially expressed (FDR $<0.05$ and a FC $>2$ (Fold Change)) between the phosphomutant groups. c Volcano plot showing genes differentially expressed when comparing S181D (upper graph) or S181A (lower graph) with S181 expressing cells. Genes with an FDR $<0.05$ and a

637 FC $>2$ are colored: red upregulated and green downregulated. The name of genes of interest is 638 indicated. d RNA extraction from DLD-1 KO $\left(\mathrm{KRAS}^{\mathrm{WT} /}\right)$ cells stably expressing KRAS-G12V- 
S181, -S181A or -S181D was carried out and cDNA was obtained from $1 \mu \mathrm{g}$ of total RNA. Real

640 Time qPCR was performed. The normalized expression of CTNNA1, SERPINE1, PRSS2, 641 HNF4G and TRIB2 is presented relative to the expression in KRAS-G12V-S181 642 phosphomutant. Data shown represent the mean \pm SEM of three independent experiments 643 (S181, S181A and S181D indicate the average of three different KRAS-G12V-S181, -S181A or 644 -S181D cell clones). Significant differences were assessed using one-way ANOVA and 645 Dunnett's Multiple Comparisons Tests compared to S181 (*p-value $<0.05$, **p-value $<0.01$, $* * *$ p-value $<0.001, * * * *$ p-value $<0.0001)$. e GSEA plot showing enrichment of the indicated gene set in the expression profile of S181D versus S181 and S181A versus S181D cells. NES, normalized enrichment score; $\mathrm{P}, \mathrm{p}$-value.

Fig. 3 Oncogenic KRAS phosphorylation/dephosphorylation cycle at Ser181 is necessary to induce an epithelial polarized structure. a Cell extracts from DLD-1 KO (KRAS ${ }^{\mathrm{WT} /}$ ) cells stably expressing KRAS phosphomutants cultured in 2D were immunoblotted using the indicated antibodies. CDK4 and lamin B were used as loading controls. b $2.5 \times 10^{4}$ DLD-1 KO $\left(\right.$ KRAS $\left.^{\mathrm{WT} /}{ }^{-}\right)$cells stably expressing either KRAS-G12V-S181, -S181A, or -S181D were cultured on top of a thin basement membrane matrix (Matrigel) overlaid with a dilute solution of this basement membrane matrix (3D on-top Matrigel assay). Representative phase-contrast images of phosphomutants cells grown for seven days are shown. All scale bars, $50 \mu \mathrm{m}$. c After seven days, colonies were immunostained to detect E-cadherin (adherent junctions, green), integrin $\alpha$ 6 (basement membrane marker, green), $\alpha$-E-catenin (cell adhesion, green) and polymerized actin was detected with phalloidin (apical cell marker, red). Nuclei were counterstained with DAPI (blue). A representative image of one of each phosphomutants is shown. All scale bars, $66210 \mu \mathrm{m}$.

Fig. 4 Oncogenic KRAS phosphorylation/dephosphorylation cycle at Ser181 regulates cell invasive capacity. SW480 cells (S181) and different clones of CRISPR modified SW480 cells with one KRAS allele with S181A mutation (named as S181A followed by the number of the 
clone) were used. a RNA extraction from SW480 cells (S181) and SW480 cells with S181A mutation was carried out and cDNA was obtained from $1 \mu \mathrm{g}$ of total RNA. Real Time qPCR was performed. The normalized expression of SERPINE1 and CTNNA1 is expressed in the graph relative to SW480 cells (S181). Data shown represent the mean \pm SEM of four independent experiments. Significant differences were assessed using one-way ANOVA and Dunnett's multiple comparisons tests compared to SW480 cell line (S181) (*p-value $<0.05$, **pvalue $<0.01, * * *$ p-value $<0.001, * * * *$ p-value $<0.0001)$. \# significant differences using unpaired two-tailed t test. b Cell extracts from SW480 cells (S181) and SW480 cells with S181A mutation were immunobloted to detect the indicated proteins. Gap120 and CDK4 were used as loading controls. c Cell invasion assay was performed as detailed in methods section. The number of invading cells was calculated as the number of cells counted in the lower compartment of Boyden chamber divided by the number of areas counted. Data show the invading cell ratio and represent the mean \pm SEM of three independent experiments. Significant differences were assessed using one-way ANOVA and Dunnett's Multiple Comparisons Tests compared to SW480 cell line (S181) $\left({ }^{*}\right.$ p-value $<0.05, * * p$-value $<0.01, * * * p$-value $<0.001$, $* * * * \mathrm{p}-$ value $<0.0001)$.

Fig. 5 Phosphorylation at Ser181 of oncogenic KRAS is necessary for tumor growth. DLD$1 \mathrm{KO}\left(\mathrm{KRAS}^{\mathrm{WT} /}\right.$ ) cells stably expressing either KRAS-G12V-S181 (clone S3), -S181A (clone A1) or - S181D (clone D2) were injected into each flank of nude mice (each group $n=4$ tumors). a Oncogenic KRAS exogenous protein levels from the different cell clones were analyzed by immunoblot the day of injection into mice. Lamin B was used as loading control. b At day 28 mice were euthanized, and tumors were dissected and weighed. The weight of excised tumors is showed in the graph (each dot corresponds to a tumor). Mean \pm SD of four tumors of each phosphomutant is shown. Significant differences were assessed using one-way ANOVA and Dunnett's Multiple Comparisons Tests comparing to S181-derived tumor (*p-value $<0.05,{ }^{*}$ pvalue $<0.01, * * *$ p-value $<0.001, * * * *$ p-value $<0.0001)$. c Histology of tumors was analyzed by hematoxylin-eosin staining. Slide scan and morphometric analysis were performed. The panels 
show from left to right the lowest to highest magnification images. Scale bars of lowest magnification, $200 \mu \mathrm{m}$. Scale bars of highest magnifications, $50 \mu \mathrm{m}$. d-e Total cell lysates of representative excised tumors were immunoblotted to detect the indicated proteins (numbers indicate different tumors). CDK4, tubulin and Gap120 were used as loading controls (d). Gap120 and *Gap120 were used as loading controls of phospho- and total proteins, respectively (e).

\section{Fig. 6 Expression of S181D and S181A signature in human CRC primary tumors and} normal colon. a Correlation matrix (Pearson's Coefficient) between the expression of genes belonging to S181A and S181D signatures analyzed in human CRC primary tumors (GSE39582). We are more restrictive with the S181D signature in order to have a similar number of genes in each one. UGT1A1-10 and CTNNA1 are excluded from the analysis (the first because is a group of genes and the second because it belongs to both signatures). Correlation is considered if $\mathrm{p}$-value $\mathrm{P}<0.01$ (student $\mathrm{T}$-test). $\mathbf{b}$ Color-map showing relative expression of genes belonging to S181A and S181D signatures in CRC human primary tumors (CRC T) versus normal tissue (NT) (GSE39582). Differences were considered if p-value $\mathrm{P}<0.01$. c DFS Kaplan-Meyer curves using the same cohort as in (a) and (b). Each curve represents the percentage ( $\mathrm{Y}$-axis) of the population that exhibits recurrence of the disease along time (X-axis, in months) for each indicated quartile.

\section{Authors' contributions}

DC and SB contributed equally to this work; DC, SB, NP, MMG, BA and NG-S conducted the experiments and data analysis; $\mathrm{CB}, \mathrm{MC}, \mathrm{JME}, \mathrm{MB}, \mathrm{CR}, \mathrm{GC}, \mathrm{MJ}$ and NA conducted data analysis and interpretation; TR-F, GP and CR provided technical set-up and support. MJ and NA designed the study; MJ, DC and NA wrote the manuscript. All authors read and approve the final manuscript.

NA (neusagell@ub.edu) and MJ (mjaumot@ub.edu) are corresponding authors. 


\section{Figure 1}

a
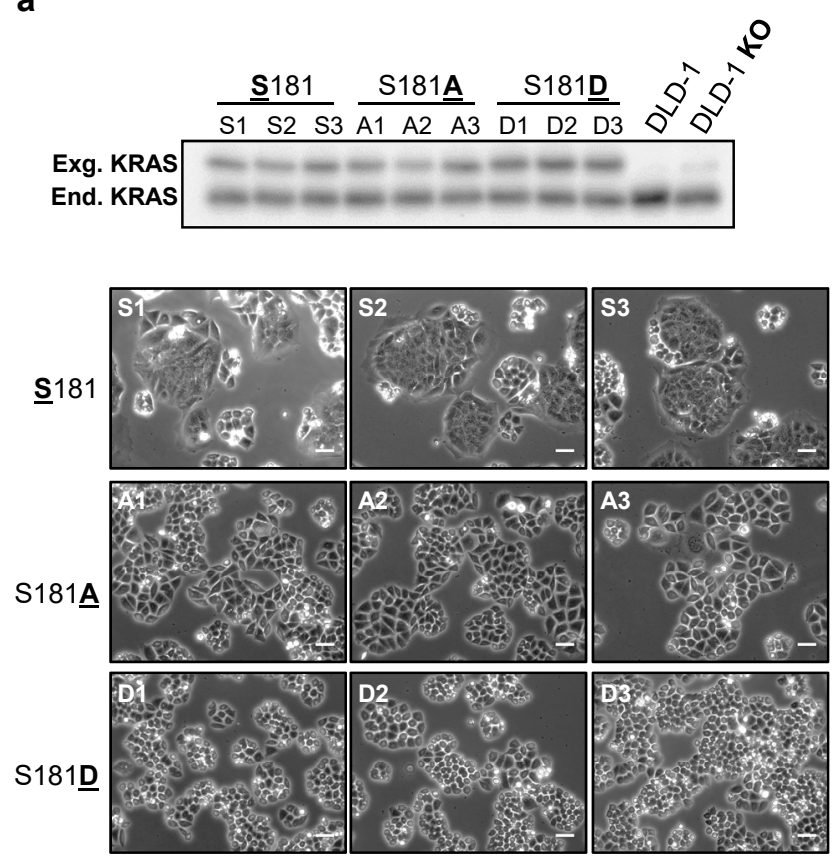

C

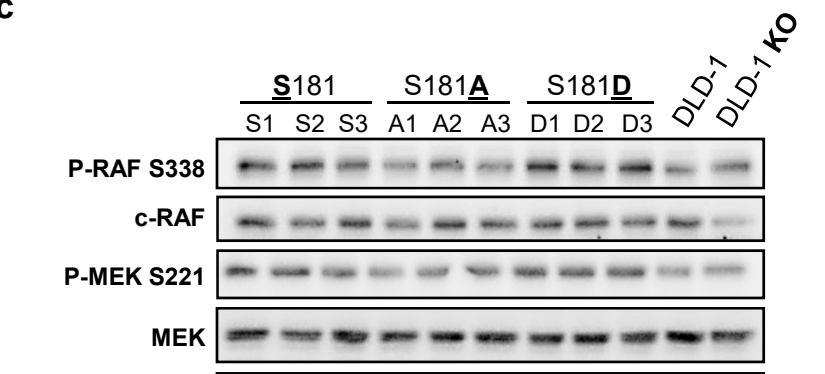

P-ERK T202/Y204 $= \pm=-= \pm= \pm=\square$

ERK1/2 $==$ = = = = = =

Exg. KRAS $-(-\cdots--$

End. KRAS $-\cdots-\cdots-\cdots$

Lamin B --------- b

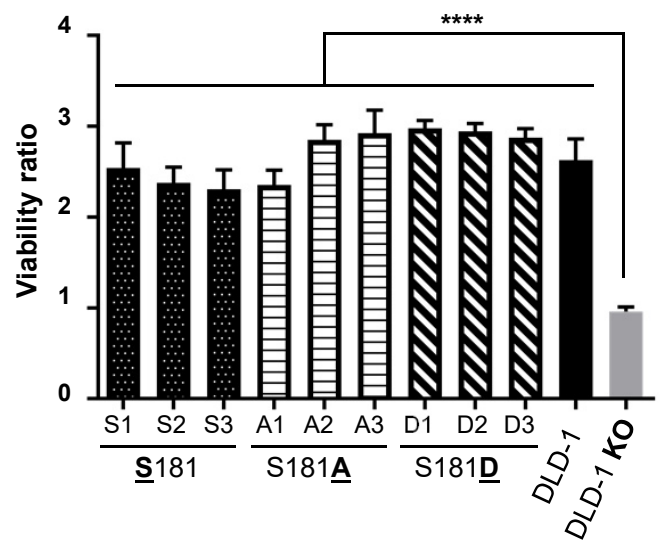


Figure 2
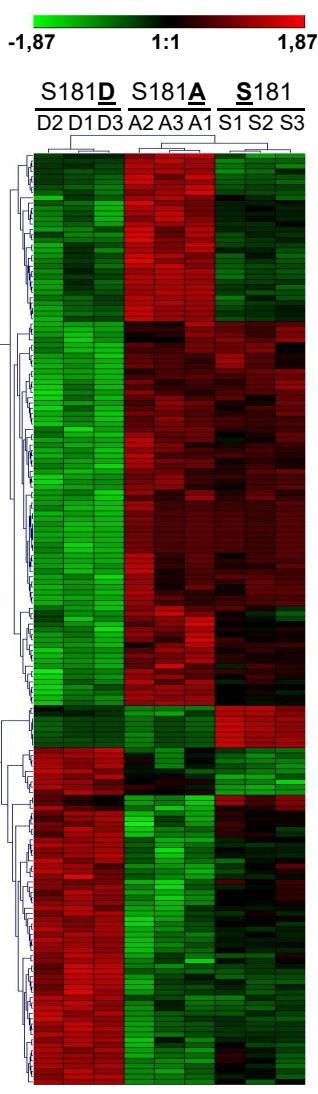

b
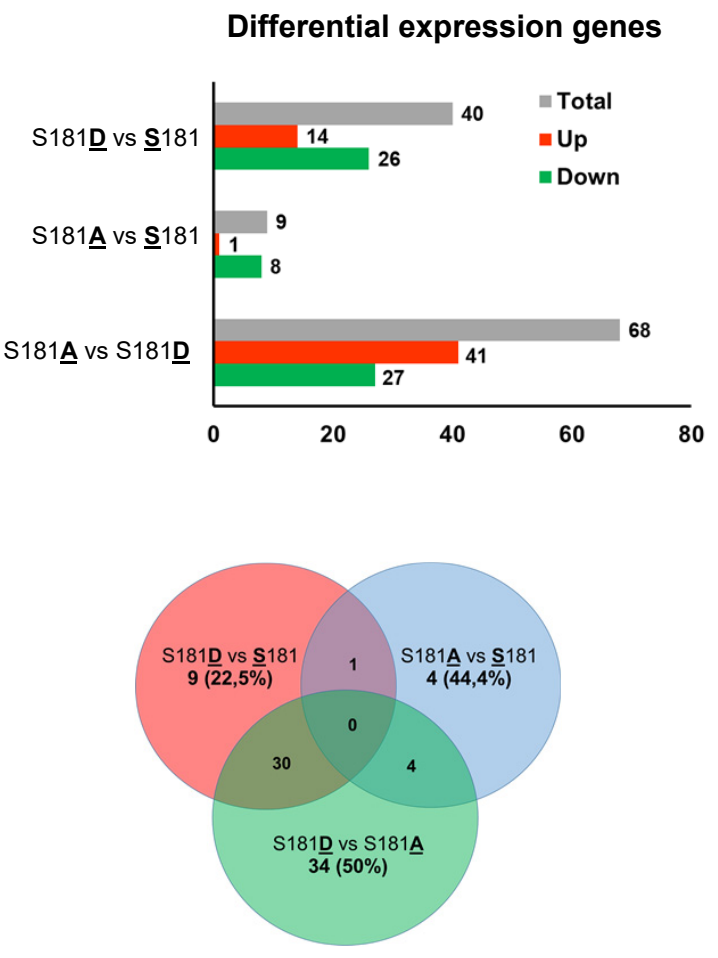

C
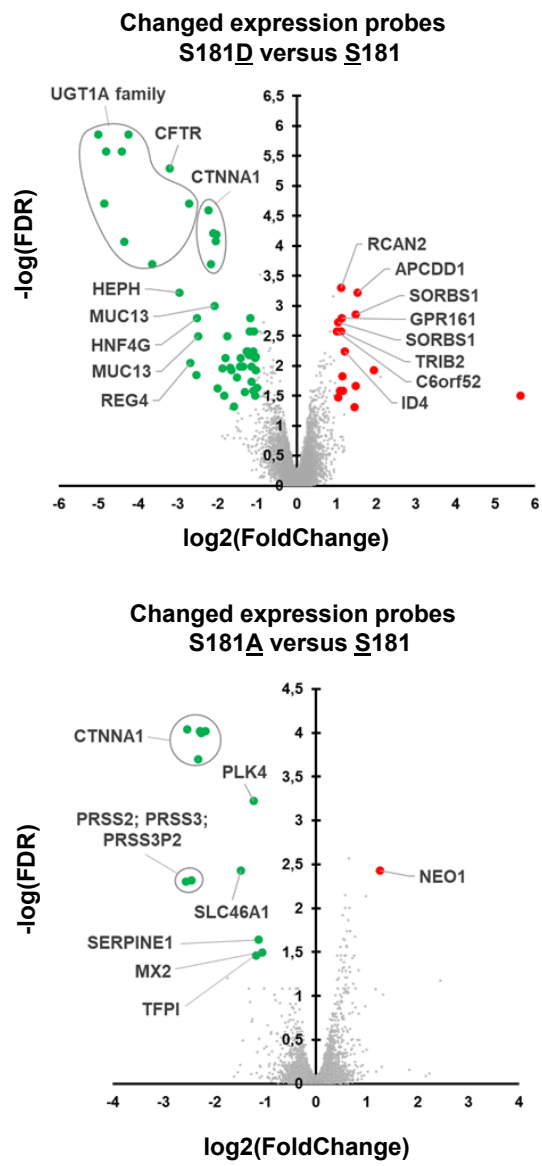

d
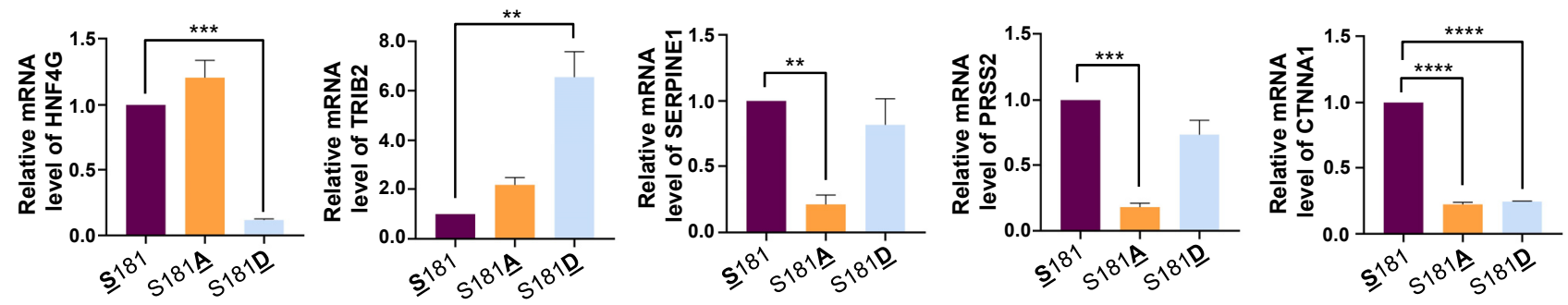

e
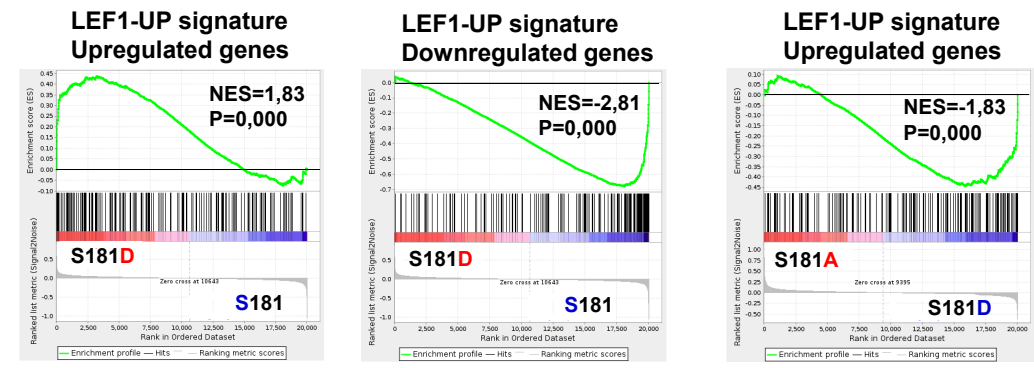

LEF1-UP signature

Downregulated genes

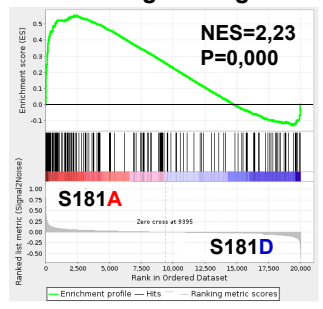




\section{Figure 3}

a

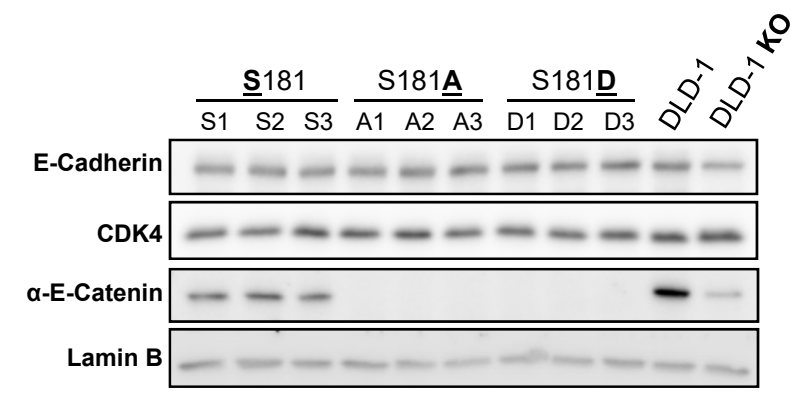

b

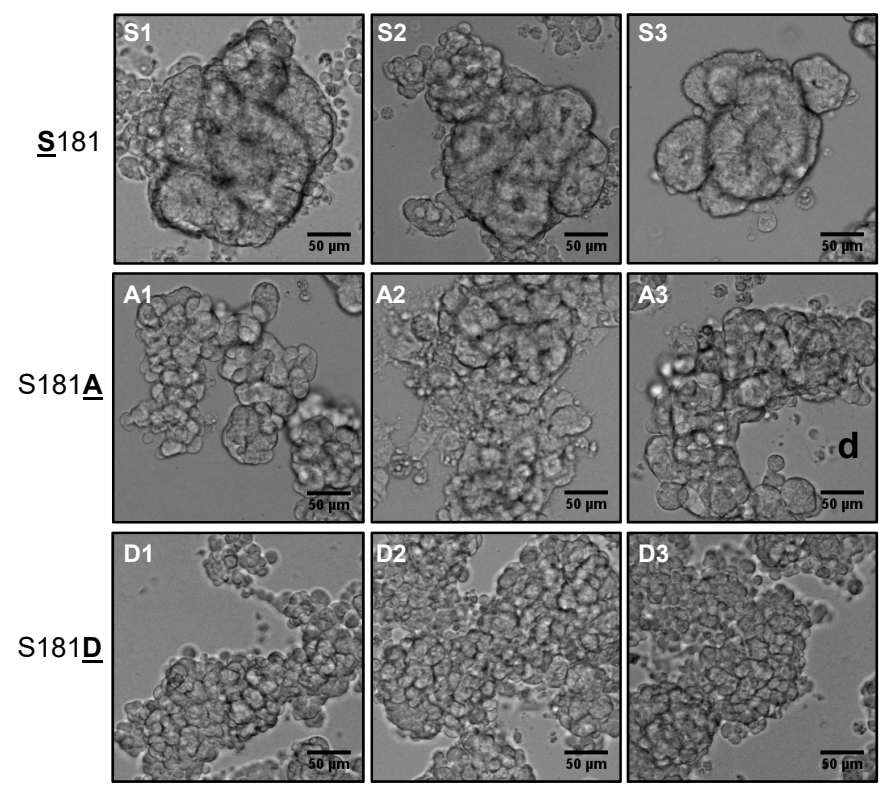

C

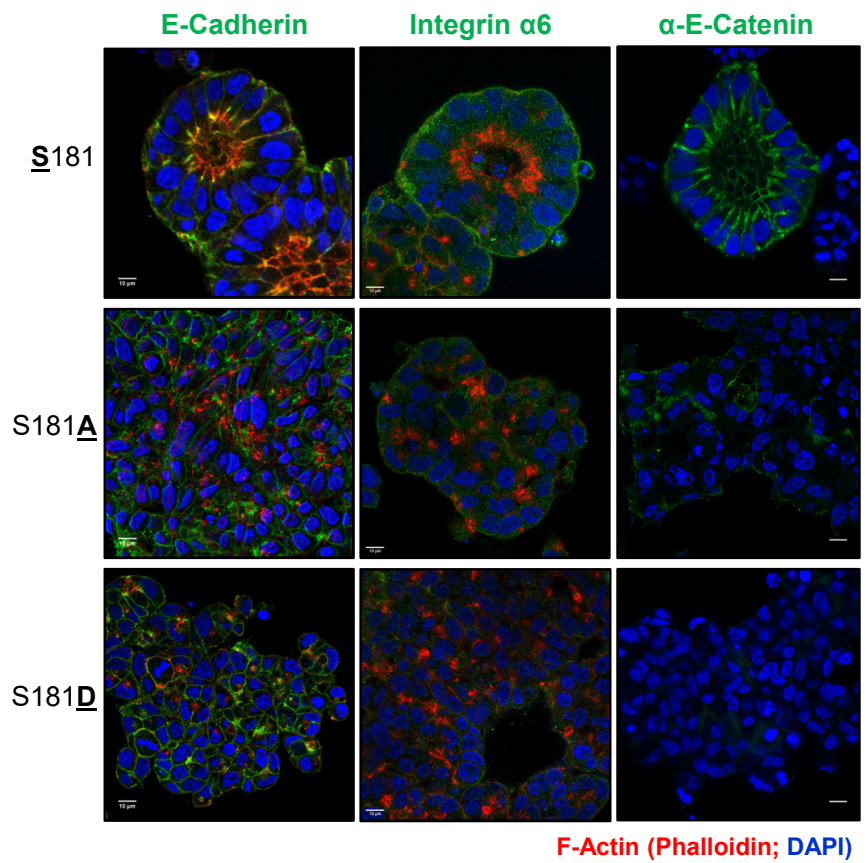




\section{Figure 4}

a
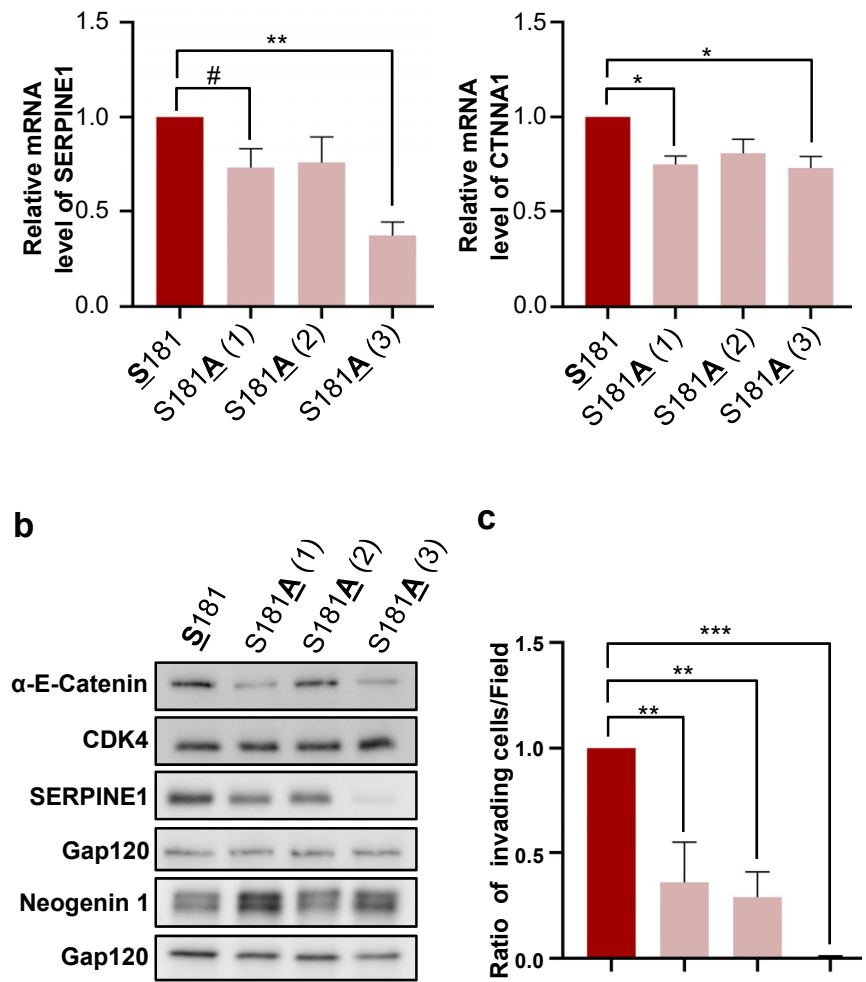

C

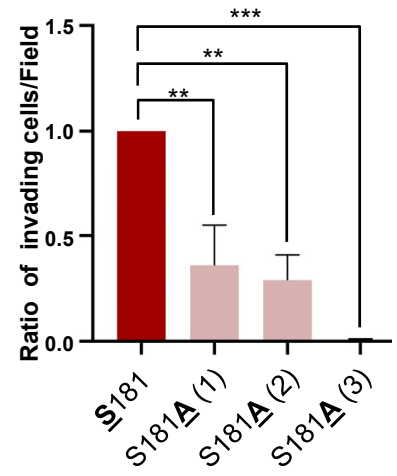




\section{Figure 5}

a

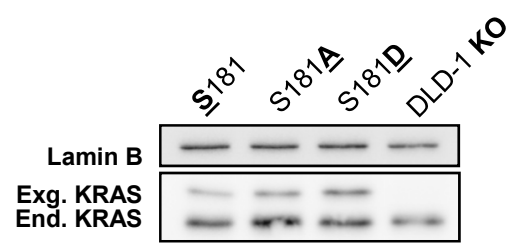

b

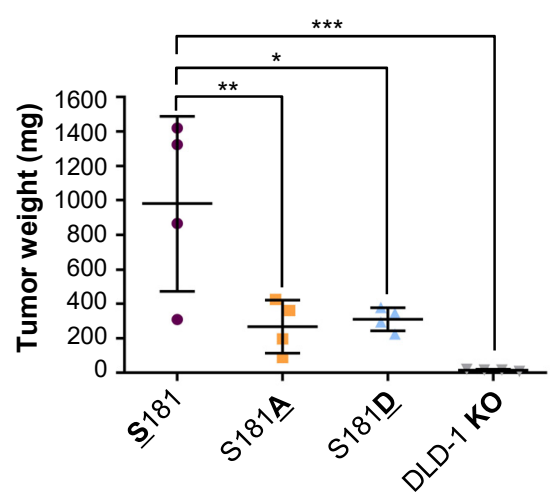

d

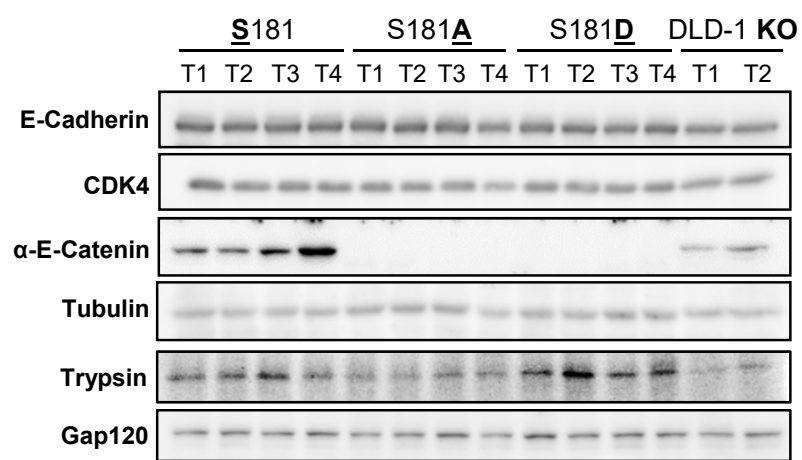

C

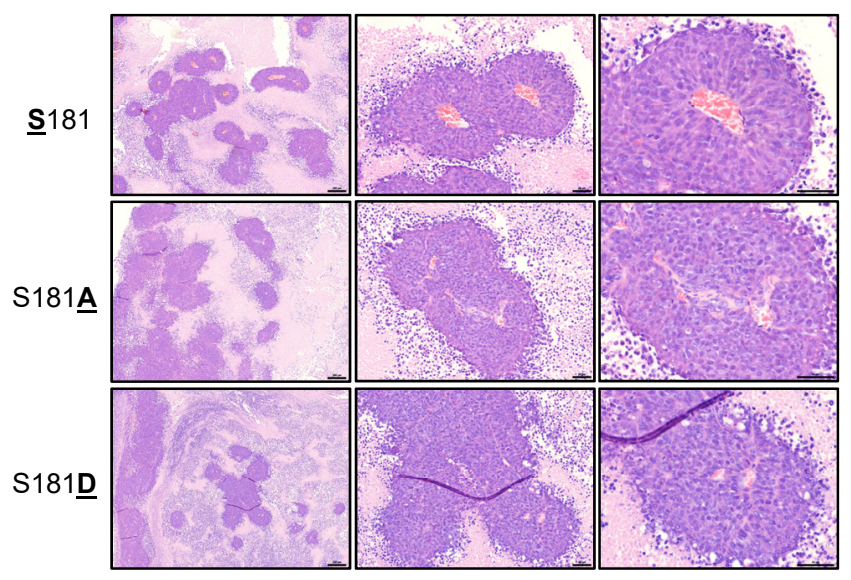

e S181 S181A S181D DLD-1 KO $\overline{\mathrm{T} 1 \mathrm{~T} 2 \mathrm{~T} 3 \mathrm{~T} 4} \overline{\mathrm{T} 1 \mathrm{~T} 2 \mathrm{~T} 3 \mathrm{~T} 4} \frac{\mathrm{T} 1 \mathrm{~T} 2 \mathrm{~T} 3 \mathrm{~T} 4}{\mathrm{~T} 1 \mathrm{~T} 2}$

\begin{tabular}{rl|} 
P-RAF S338 & \\
C-RAF & \\
ERK & \\
P-ERK T202/Y204 & \\
Exg. KRAS \\
End. KRAS \\
Gap120 \\
Gap120* \\
G
\end{tabular}

P-AKT S473 $-1-1-1-1-\cdots$ Gap120

АKT $---\cdots-\cdots-\cdots$ Gap120* 
Figure 6

a

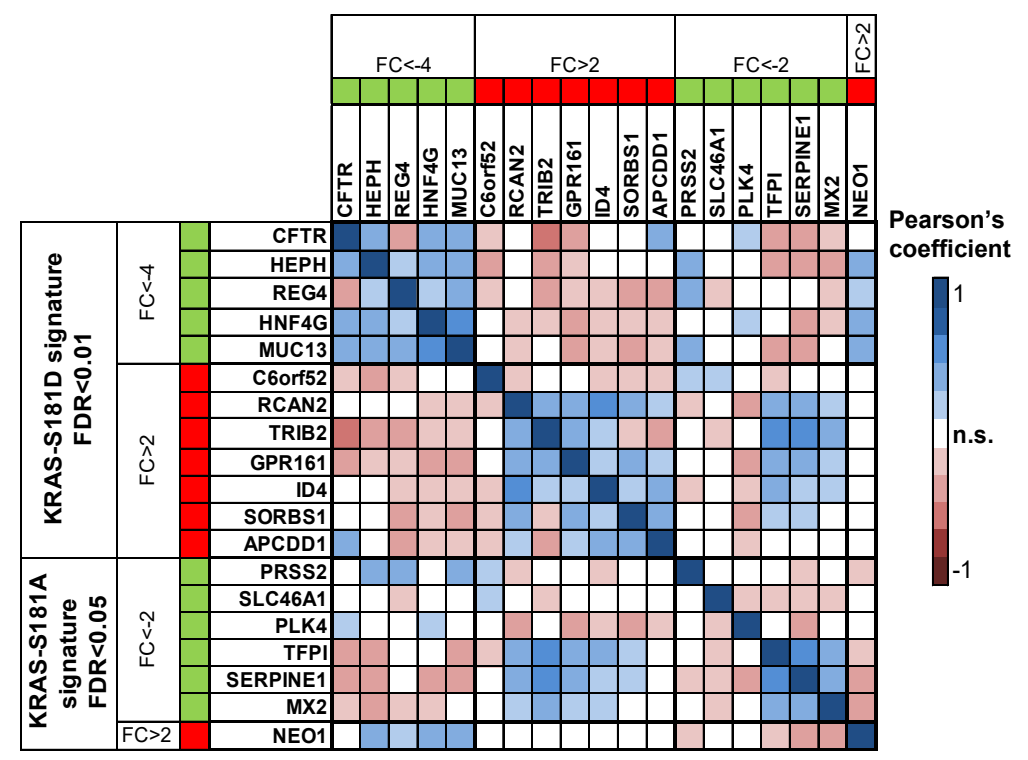

b
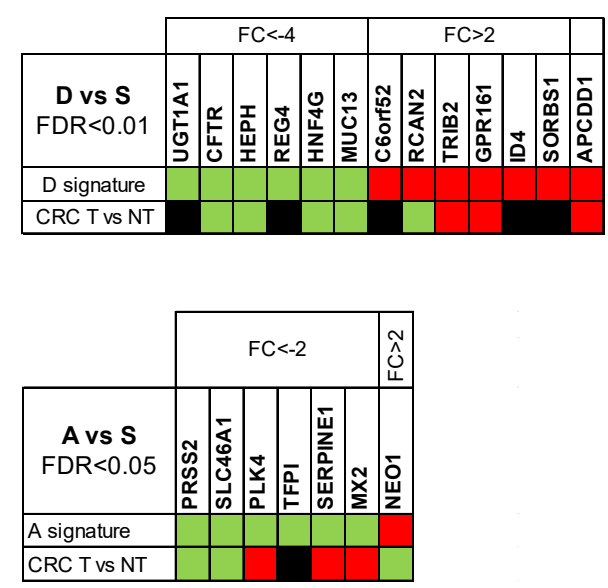

up down n.s.

C

HNF4G

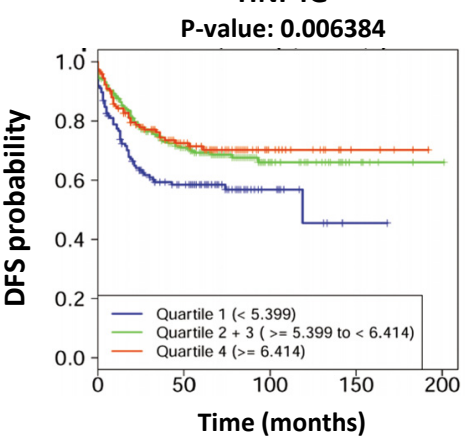

ID4

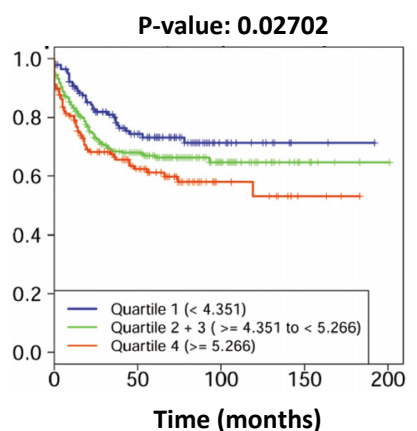

SERPINE1

P-value: $\mathbf{0 . 0 0 1 1 8 5}$

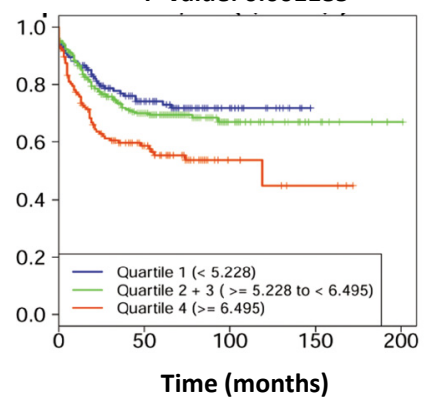

NEO1

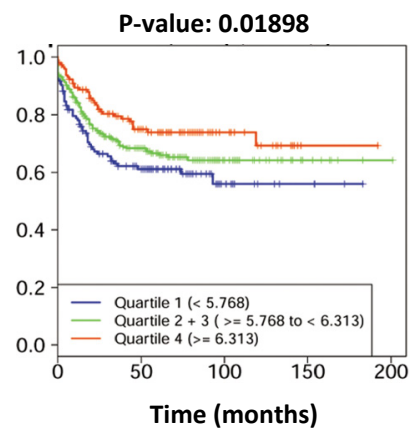

